\title{
Atividade moluscicida de princípios ativos de folhas de Lycopersicon esculentum (Solanales, Solanaceae) em Biomphalaria glabrata (Gastropoda, Planorbidae)
}

\author{
Vilma Leyton $^{1}$, Thomas O. Henderson ${ }^{2}$, Douglas Mascara ${ }^{3} \&$ Toshie Kawano $^{4}$ \\ 1. Depto. de Medicina Legal, Ética Médica, Medicina Social e do Trabalho, Faculdade de Medicina, Universidade de São Paulo, Rua \\ Teodoro Sampaio, 151, 05405-000 São Paulo, SP, Brasil. (vileyton@usp.br) \\ 2. Dept. of Biochemistry and Molecular Genetics, University of Illinois, 900 S. Ashland Ave. (m/c 669), Chicago, IL 60607, USA \\ (revtotis@mailserv.uic.edu) \\ 3. Centro de Ciências da Saúde, Universidade de Mogi das Cruzes, Av. Dr. Cândido Xavier de Almeida e Souza, 200, 087800-911 Mogi das \\ Cruzes, SP, Brasil. (dmascara@umc.br) \\ 4. Laboratório de Parasitologia, Instituto Butantan, Av. Vital Brasil, 1500, 05503-900 São Paulo, SP, Brasil. (toshie@butantan.gov.br)
}

\begin{abstract}
Moluscicide activity of active principles in the leaves of Lycopersicon esculentum (Solanales, Solanaceae) on Biomphalaria glabrata (Gastropoda, Planorbidae). Aqueous and alcoholic extracts were obtained from crushed dried leaves of tomato plant (Lycopersicon esculentum, Mill.) c.v. Cherry. By the use of a methanolic extraction and alkaline precipitation, a product named crude steroidal glycoalkaloid (GEb), was obtained. The presence of tomatidine was characterized in this product. In laboratory, the aqueous and alcoholic extracts and GEb have shown molluscicidal activity against Biomphalaria glabrata (Say, 1818). The crude steroidal glycoalkaloid presented a high molluscicidal activity $\left(\mathrm{LC}_{50}=8.01 \mathrm{ppm}\right.$ and $\left.\mathrm{LC}_{90}=13.17 \mathrm{ppm}\right)$, similar to that of tomatine. None of the compounds tested affected B. glabrata egg masses. The level of activity showed by the different extracts and by the GEb, pointed out the GEb as the only candidate able to be considered for further tests toward field trials as molluscicidal agent.
\end{abstract}

KEYWORDS. Moluscicide, Biomphalaria glabrata, Lycopersicon esculentum, tomato.

RESUMO. Foram obtidos extratos aquosos e alcoólicos a partir de pó de folhas secas de tomateiro (Lycopersicon esculentum, Mill.) c.v. Cereja. Por extração metanólica e precipitação alcalina, foi obtido um produto que denominamos "glicoalcalóide esteroidal bruto" (GEb), no qual foi caracterizada a presença de tomatina. Em ensaios laboratoriais, os extratos aquosos, alcoólicos e o GEb apresentaram atividade moluscicida em Biomphalaria glabrata (Say, 1818). O "glicoalcalóide esteroidal bruto" apresentou alta atividade moluscicida (CL50 = $8,01 \mathrm{ppm}$ e CL90 = 13,17 ppm), comparável à atividade da tomatina. Desovas de B. glabrata mostraram-se resistentes aos extratos testados. Os níveis de atividade moluscicida apresentados pelos diversos extratos e o GEb, apontam apenas o GEb como candidato para a continuação dos estudos visando a sua possível utilização em campo.

PALAVRAS-CHAVE. Moluscicida, Biomphalaria glabrata, Lycopersicon esculentum, tomateiro.

Moluscicidas são substâncias usadas para exterminar caramujos que vivem e se alimentam de folhagens nos jardins, lavouras, estufas e campos. São também utilizados para controlar caramujos vetores de parasitas importantes em saúde pública (WARE, 1978; OMS, 1991). Os programas de controle que incluem o uso de moluscicidas estão voltados para o combate a moluscos transmissores da esquistossomose e de moléstias como a fasciolose, causada por Fasciola hepatica (Linnaeus, 1758) (Trematoda, Digenea) e que tem como hospedeiro intermediário Lymnaea spp. (Gastropoda) (GUIMARÃES, 1991).

Os moluscicidas sintéticos são utilizados em programas de controle da esquistossomose com o objetivo de combater os caramujos vetores. A preocupação com o desenvolvimento de resistência dos caramujos a essas substâncias, a baixa seletividade que apresentam (atuando sobre outras espécies da fauna, podendo perturbar o equilíbrio ecológico local) e a procura de substâncias facilmente biodegradáveis têm aumentado o interesse pelo uso de moluscicidas de origem vegetal (McCullough et al., 1980). Várias plantas e seus princípios ativos foram estudados quanto ao potencial moluscicida (Kloos \& McCullough, 1981, 1982; Lemma \& YAU, 1974).
O interesse pelo uso de moluscicidas de origem vegetal no controle da esquistossomose data da década de 1930, quando foi sugerido o plantio de Balanites aegyptiaca L. (Balanitaceae), uma árvore típica do deserto, nas margens dos focos de transmissão, no Sudão. Seus frutos, ao caírem das árvores, inibiam a densidade populacional de caramujos (ARCHIBALD, 1933). No Brasil, os primeiros estudos sobre moluscicidas de origem vegetal demonstraram a atividade de extratos aquosos de caules de Serjania spp. (cipó-timbó) e de frutos de Sapindus saponaria L. (Sapindaceae) (saboneteira, sabão) em Biomphalaria glabrata (Say, 1818). Essa ação foi atribuída às saponinas presentes nestes vegetais (Pinto \& Almeida, 1944). Mais de mil espécies vegetais foram testadas quanto à sua atividade moluscicida (AHMED et al., 1984; FARNSWORTH et al., 1987). No Brasil, várias plantas medicinais, tóxicas e ornamentais foram testadas com a finalidade de verificar sua possível ação moluscicida (AMORIN \& Pessoa, 1962; Mendes et al., 1984; Rouquayrol et al., 1972; SiLVA et al., 1971).

Visando testar sua atividade moluscicida, foram utilizadas folhas de tomateiro como matéria prima para obtenção de substâncias a serem empregadas no controle de B. glabrata. 


\section{MATERIALEMÉTODOS}

A planta utilizada foi Lycopersicon esculentum Mill. (Solanaceae), cv Cereja (tomateiro), coletada no período de frutificação (1995). Exsicatas foram depositadas e catalogadas (SPF 88.585) no herbário do Departamento de Botânica, Instituto de Biociências, Universidade de São Paulo.

Os caramujos utilizados nos testes foram espécimens de B. glabrata (Say, 1818) (Gastropoda), linhagem selvagem, mantidos por várias gerações no laboratório de Malacologia do Instituto Butantan. Esses animais descendem de exemplares provenientes de Belo Horizonte, Barreiro de Baixo, Minas Gerais, adaptados às condições do laboratório (temperatura de $20-25^{\circ} \mathrm{C}$ e regime de iluminação natural), em aquários de polietileno ( $22 \mathrm{x}$ $55 \times 17 \mathrm{~cm}$ ) com cerca de 20 litros de água filtrada mantida em repouso por um período mínimo de 24 horas para a eliminação do cloro. Os aquários foram arejados por meio de bombas de ar de $1,5 \mathrm{~W}$ e a troca de água era realizada a cada 15 dias. $\mathrm{O}$ alimento fornecido ad libitum constituiuse de alface fresca ou seca em estufa. Foram utilizados espécimens adultos, selecionados ao acaso, medindo entre 11 e 18 mm de diâmetro. As desovas utilizadas foram coletadas desta mesma população de caramujos.

A substância comercial Bayluscide na forma de pó molhável, contendo $70 \%$ de sal de etanolamina da niclosamida, foi utilizada nos ensaios biológicos como moluscicida de referência. A tomatina e tomatidina foram obtidas comercialmente.

Pó de folhas secas de tomateiro (dessecadas em estufa a $55^{\circ} \mathrm{C}$ ) foi utilizado para o preparo de extratos aquosos "a frio" (AQf) e "a quente" (AQq), etanólico, metanólico e um produto denominado glicoalcalóide esteroidal bruto $(\mathrm{GEb})$.

Para o preparo do extrato aquoso "a frio" (AQf), o pó de folhas secas foi submetido a agitação (agitador magnético) por quatro horas com água destilada à temperatura ambiente, filtrado com auxílio de funil tipo "Buchner" e o volume ajustado com água destilada para obtenção de extrato de concentração 10.000 ppm. Para o preparo do extrato aquoso "a quente" (AQq), o material vegetal foi submetido à fervura (decocção) com água por cinco minutos e o volume do extrato obtido, filtrado ainda quente, foi ajustado com água em quantidade suficiente para se obter solução de concentração 10.000 ppm.

O extrato etanólico foi obtido a partir da extração do material vegetal com etanol p.a. em aparelho de refluxo por uma hora. O extrato foi filtrado e evaporado, sob pressão reduzida em equipamento "rotavapor", até a remoção completa do solvente. Para obtenção do extrato metanólico, o material vegetal foi submetido à fervura em aparelho de refluxo, durante quinze minutos, quatro vezes consecutivas, utilizando volumes de $200 \mathrm{ml}$ de metanol. Os extratos combinados foram evaporados sob pressão reduzida até a remoção completa do solvente. Os resíduos obtidos nestas extrações foram dissolvidos com os respectivos álcoois para obtenção de extratos de concentração 200.000 ppm.

A partir do GEb, utilizando método descrito por FontainE et al. (1948), que consiste em solubilizações e reprecipitações sucessivas com ácido clorídrico e hidróxido de amônio, o material vegetal foi extraído em aparelho de refluxo com $200 \mathrm{ml}$ de metanol por quinze minutos e o extrato obtido filtrado através de papel de filtro. Este procedimento foi repetido quatro vezes e os extratos combinados foram evaporados em banho de água com temperatura inferior a $45^{\circ} \mathrm{C}$. Ao balão contendo o resíduo, foram adicionados $300 \mathrm{ml}$ de água destilada e a mistura submetida à fervura durante uma hora. A solução resultante foi filtrada e, após resfriamento, alcalinizada a $\mathrm{pH} 10,0$ com $\mathrm{NH}_{4} \mathrm{OH} 10 \%$. A solução sobrenadante foi sifonada e descartada. O precipitado formado foi ressuspendido em água e o $\mathrm{pH}$ ajustado para $4,0 \mathrm{com} \mathrm{HCl}$ $10 \%$. Essa acidificação levou à dissolução do precipitado, obtendo-se uma solução clara. Novamente, nesta solução foi adicionado $\mathrm{NH}_{4} \mathrm{OH}$ até $\mathrm{pH} 10,0$, ocorrendo novamente precipitação. Esse processo foi repetido mais duas vezes. O precipitado final foi recolhido por centrifugação e seco por evaporação. O resíduo foi dissolvido em quantidade suficiente de metanol para se obter uma solução de concentração 10.000 ppm.

Os ensaios biológicos foram realizados com fragmentos de folhas frescas, pó obtido de folhas secas, extratos aquosos e alcoólicos, GEb, tomatina e tomatidina. Em todos os ensaios foram introduzidos grupos controle utilizando água e soluções de etanol e metanol de concentração igual às usadas nos testes. A cada duas semanas, foram realizados ensaios com a substância comercial Bayluscide como moluscicida de referência.

A verificação da atividade moluscicida em espécimens adultos de $B$. glabrata seguiu os procedimentos recomendados pela WHO (1965). Dez grupos de cinco animais foram transferidos para miniaquários preenchidos com as soluções a serem testadas e cobertos com placas de Petri. Após um período de exposição de 24 horas, os animais foram retirados, lavados cinco vezes com água e transferidos para copos de plástico contendo água filtrada, onde permaneceram por um período de recuperação de mais 24 horas, quando a mortalidade foi registrada. Como critério de morte, foi adotada a retração dos caramujos para dentro de suas conchas ou a liberação de hemolinfa.

As desovas de B. glabrata foram coletadas em tiras de polietileno incolor colocadas na superfície da água dos aquários na tarde anterior do dia do experimento. As regiões do plástico com as desovas foram recortadas, transferidas para placas de Petri contendo água e observadas em microscópio esteroscópico para a seleção. Foram utilizadas aquelas que possuíam membranas intactas. As desovas (aproximadamente 20-30 ovos), no estágio de blástula (5-15 horas depois da primeira divisão do ovo), foram transferidas, em dez grupos de três, para placas de Petri contendo $10 \mathrm{ml}$ de soluções preparadas a partir dos extratos, do GEb e dos padrões tomatina e tomatidina. Após um período de 24 horas de exposição, as desovas foram lavadas e transferidas para placas de Petri contendo água filtrada e declorada. Após dez dias foi verificada a ocorrência ou não de eclosão.

As atividades moluscicidas, expressas em concentrações letais de $50 \%$ e $90 \%\left(\mathrm{CL}_{50}\right.$ e $\left.\mathrm{CL}_{90}\right)$ foram determinadas a partir dos dados obtidos nos ensaios biológicos com espécimens adultos de B. glabrata. Estas concentrações foram analisadas pelo método dos 
probitos no programa PROBITCF em linguagem BASIC, desenvolvido pelo Prof. Dr. Paulo A. Otto do Departamento de Biologia/Genética, Instituto de Biociências, USP, São Paulo. A comparação entre os dados das $\mathrm{CL}_{50}$ e $\mathrm{CL}_{90}$ dos extratos aquoso "a frio" e "a quente" e a dos extratos etanólico e metanólico foi realizada utilizando o teste Qui-quadrado $\left(\chi^{2}\right)$, considerando o nível de confiança $\alpha=0,05$.

\section{RESULTADOS E DISCUSSÃO}

O extrato aquoso "a frio" apresentou $\mathrm{CL}_{50}=210,39$ ppm e $\mathrm{CL}_{90}=376,12$ ppm e o extrato aquoso "a quente", $\mathrm{CL}_{50}=216,91 \mathrm{ppm}$ e $\mathrm{CL}_{90}=408,98 \mathrm{ppm}$ (tab. I). Observando-se estes resultados, percebe-se que não houve diferença significativa $\left(\chi^{2}=0,197 ; \mathrm{P}=0,657\right)$ entre a atividade moluscicida destes dois extratos quando testados em $B$. glabrata.

Para o extrato etanólico, as concentrações letais determinadas foram: $\mathrm{CL}_{50}=74,35 \mathrm{ppm}$ e $\mathrm{CL}_{90}=185,60$ ppm e para o extrato metanólico, $\mathrm{CL}_{50}=94,31 \mathrm{ppm} \mathrm{e} \mathrm{CL}_{90}=$ 197,30 ppm (tab. I). Neste caso, também não houve diferença significativa da atividade moluscicida entre os dois extratos $\left(\chi^{2}=0,899 ; \mathrm{P}=0,343\right)$. Estes resultados sugerem que os princípios ativos responsáveis pela ação moluscicida são extraídos do pó de folhas secas em quantidades semelhantes tanto com etanol como com metanol.

Comparando-se as concentrações letais, obtidas com extratos aquosos e alcoólicos, pode-se verificar que estes últimos foram aproximadamente três vezes mais eficazes para caramujos adultos de B. glabrata do que os extratos aquosos "a frio" ou "a quente". Através dos dados dos ensaios realizados com o $\mathrm{GEb}$ obteve-se $\mathrm{CL}_{50}=$ $8,01 \mathrm{ppm}$ e $\mathrm{CL}_{90}=13,17 \mathrm{ppm}$ (tab. I). As $\mathrm{CL}_{50}$ e $\mathrm{CL}_{90}$ determinadas para a tomatina foram 4,49 ppm e 11,84 ppm, respectivamente (tab. I). Estes resultados mostram que essas substâncias são altamente eficazes em B. glabrata. HostetTMAnN et al. (1982) apontaram que a tomatina obtida comercialmente era ativa a $4 \mathrm{ppm}(100 \%$ de mortalidade) quando testada em B. glabrata. AlzérReCA $\&$ HART (1982) obtiveram 80\% de mortalidade com 25 ppm de tomatina comercial, também com B. glabrata.

Ensaios realizados com a tomatidina (grupamento aglicona da tomatina) obtida comercialmente mostraram que esta substância não é eficaz em concentrações até 25 ppm para espécimens adultos de B. glabrata. Esta resposta já foi observada por AlzérReCA \& HART (1982) não só para $B$. glabrata como também para Lymnaea cubensis (Pfeiffer, 1839). Neste mesmo estudo, os autores também avaliaram os efeitos tóxicos dos glicoalcalóides esteroidais, solasonina e solamargina, extraídos da Solanum mammosum L. (Solanaceae), bem como a solasodina, grupamento aglicona daqueles glicoalcalóides, em B. glabrata e L. cubensis. Os resultados obtidos mostraram que somente a solasonina e a solamargina foram eficazes frente aos animais testados, enquanto que a solasodina não apresentou atividade moluscicida. Estes resultados sugerem que a presença de açúcares nas moléculas dos glicoalcalóides esteroidais parece ser essencial para a ação moluscicida.
Os resultados da exposição de B. glabrata ao Bayluscide por 24 horas, seguidas de igual período de recuperação, permitiram calcular a $\mathrm{CL}_{50} \mathrm{em} 0,029 \mathrm{ppm}$ e a $\mathrm{CL}_{90}$ em 0,087 ppm.

Soluções de concentração até 1.000 ppm, obtidas a partir dos extratos aquosos "a quente" e "a frio", não foram eficazes em desovas de $B$. glabrata que eclodiram no tempo esperado ( 9 a 10 dias após a oviposição). O mesmo ocorreu com as soluções de concentração até 200 ppm preparadas a partir dos extratos etanólico e metanólico. O GEb e os padrões de tomatina e tomatidina não apresentaram atividade em concentração até $50 \mathrm{ppm}$.

A baixa suscetibilidade de desovas também foi relatada por RouQuAYrol et al. (1973), que demonstraram

Tabela I. Atividade moluscicida dos extratos aquosos, etanólico e metanólico de folhas de Lycopersicon esculentum, do glicoalcalóide esteroidal bruto (Geb) e tomatina em Biomphalaria glabrata (Teste Probit-Log) com tempo de exposição e de recuperação de 24 horas (AQf, extrato aquoso "frio"; AQq, extrato aquoso "quente"; C, concentração; CL, concentração letal; exp, expostos; mor, mortos).

\begin{tabular}{|c|c|c|c|c|}
\hline \multirow[t]{2}{*}{ Extratos } & \multirow{2}{*}{$\begin{array}{c}\mathrm{C} \\
(\mathrm{ppm})\end{array}$} & \multicolumn{2}{|c|}{$\mathrm{N}^{\circ}$ de animais } & \multirow{2}{*}{$\begin{array}{c}\text { Valores de CL(ppm) } \\
\text { (limites de confiança 95\%) }\end{array}$} \\
\hline & & $\exp$ & mor & \\
\hline \multirow{6}{*}{ AQf } & 100 & 50 & 1 & \multirow{6}{*}{$\begin{array}{l}\mathrm{CL}_{50}=210,39 \\
(192,04-230,50) \\
\mathrm{CL}_{90}=376,12 \\
(327,98-431,33)\end{array}$} \\
\hline & 200 & 50 & 32 & \\
\hline & 250 & 50 & 36 & \\
\hline & 300 & 50 & 42 & \\
\hline & 500 & 50 & 46 & \\
\hline & 1000 & 50 & 50 & \\
\hline \multirow{7}{*}{$\mathrm{AQq}$} & 100 & 50 & 4 & \multirow{7}{*}{$\begin{array}{l}\mathrm{CL}_{50}=216,91 \\
(197,72-237,95) \\
\mathrm{CL}_{90}=408,98 \\
(361,84-462,25)\end{array}$} \\
\hline & 200 & 50 & 22 & \\
\hline & 250 & 50 & 27 & \\
\hline & 300 & 50 & 39 & \\
\hline & 400 & 50 & 44 & \\
\hline & 500 & 50 & 47 & \\
\hline & 750 & 50 & 50 & \\
\hline \multirow{7}{*}{ Etanólico } & 10 & 50 & 0 & \multirow{7}{*}{$\begin{array}{l}\quad \mathrm{CL}_{50}=74,35 \\
(66,46-83,17) \\
\mathrm{CL}_{90}=185,60 \\
(149,90-229,80)\end{array}$} \\
\hline & 25 & 50 & 5 & \\
\hline & 37,5 & 50 & 10 & \\
\hline & 50 & 50 & 12 & \\
\hline & 75 & 50 & 20 & \\
\hline & 100 & 50 & 29 & \\
\hline & 150 & 50 & 50 & \\
\hline \multirow{7}{*}{ Metanólico } & 25 & 50 & 2 & \multirow{7}{*}{$\begin{array}{l}\mathrm{CL}_{50}=94,31 \\
(85,91-103,54) \\
C L_{90}=197,30 \\
(171,19-227,40)\end{array}$} \\
\hline & 50 & 50 & 7 & \\
\hline & 75 & 50 & 16 & \\
\hline & 100 & 50 & 22 & \\
\hline & 150 & 50 & 38 & \\
\hline & 200 & 50 & 47 & \\
\hline & 250 & 50 & 50 & \\
\hline \multirow{6}{*}{ Geb } & 5 & 50 & 4 & \multirow{6}{*}{$\begin{array}{l}\mathrm{CL}_{50}=8,01 \mathrm{Geb} \\
(7,42-8,65) \\
\mathrm{CL}_{90}=13,17 \\
(11,89-14,58)\end{array}$} \\
\hline & 7,5 & 50 & 26 & \\
\hline & 10 & 50 & 36 & \\
\hline & 12,5 & 50 & 40 & \\
\hline & 15 & 50 & 48 & \\
\hline & 20 & 50 & 50 & \\
\hline \multirow{8}{*}{ Tomatina } & 0,1 & 50 & 0 & \multirow{8}{*}{$\begin{array}{l}\mathrm{CL}_{50}=4,49 \\
(3,94-5,12) \\
\mathrm{CL}_{90}=11,84 \\
(9,59-14,62)\end{array}$} \\
\hline & 1 & 50 & 3 & \\
\hline & 2,5 & 50 & 8 & \\
\hline & 5 & 50 & 25 & \\
\hline & 7,5 & 50 & 34 & \\
\hline & 10 & 50 & 50 & \\
\hline & 10 & 50 & 0 & \\
\hline & 25 & 50 & 5 & \\
\hline
\end{tabular}


que extratos orgânicos obtidos de estruturas da Pithecelobium multiflorum Bent (Fabaceae) eram menos ativos em desovas do que em espécimens adultos de B. glabrata. LeMMA et al. (1972), em estudos realizados com frutos de Phytolacca dodecandra L'Herit (Phytolaccaceae) ("endod") secos e triturados, e extratos butílicos preparados com estes frutos, verificaram a resistência das desovas dos planorbídeos $B$. glabrata, B. alexandrina (Ehrenberg, 1831) e Bulinus truncatus (Audouin, 1827), quando expostas a concentrações que foram ativas em caramujos adultos. PEREIRA et al. (1978) obtiveram extratos hexânicos a partir de folhas de Euphorbia cotinifolia L. (Euphorbiaceae) que se mostraram ativos em desovas de B. glabrata em concentrações aproximadamente 20 vezes superiores àquelas eficazes em caramujos adultos (2,4 ppm para adultos e 48 ppm para desovas). A baixa suscetibilidade das desovas aos moluscicidas é devida, provavelmente, aos altos pesos moleculares dessas substâncias, impedindo sua penetração através da membrana gelatinosa das desovas (LEMMA \& YAU, 1974).

De acordo com recomendações da WHO (1983), somente extratos aquosos "a frio" e/ou "a quente" e alcoólicos de materiais vegetais que promovem a morte de $90 \%$ dos animais quando testados em concentrações iguais ou inferiores a 20 ppm (exposição 24 h) devem ser submetidos a ensaios em campo. As concentrações letais de $90 \%$, determinadas neste estudo, para os extratos aquosos "a frio" e "a quente", etanólico e metanólico, são superiores às estabelecidas pela WHO, implicando que estes extratos não são viáveis para estes tipos de ensaio. $\mathrm{O}$ GEb obtido neste estudo mostrou-se um moluscicida altamente eficaz quando testado em $B$. glabrata, apresentando $\mathrm{CL}_{90}=13,17$ ppm sendo, então, recomendado para futuros ensaios em campo.

\section{REFERÊNCIASBIBLIOGRÁFICAS}

Ahmed, H. M.; Bashir, A. K. \& El Kheir, Y. M. 1984. Investigations of molluscicidal activity of certain Sudanese plants used in folk-medicine. Part IV. Planta Medica 50:74-77.

Alzérreca, A. \& Hart, G. 1982. Molluscicidal steroid glycoalkaloids possessing stereoisomeric spirosalane structures. Toxicology Letters 12:151-155.

Amorin, J. P. \& PessoA, S. B. 1962. Experiência de alguns vegetais como moluscocida. Revista Brasileira de Malariologia e Doenças Tropicais 14:254-260.
Archibald, R. G. 1933. The use of the fruit of the tree Balanites aegyptiaca in the control of schistosomiasis in the Sudan. Transactions of the Royal Society of Tropical Medicine and Hygiene 27:207-210.

Farnsworth, N. R.; Henderson, T. O. \& Soejarto, D. D. 1987. Plants with potential molluscicidal activity. In: Мотт, K. E. ed. Plant molluscicides. New York, Wiley. p.131-204

Fontaine, T. D.; Irving, G. W. \& Doolittle, S. P. 1948. Isolation and partial characterization of crystalline tomatine, an antibiotic agent from the tomato plant. Archives of Biochemistry 18:467-475.

Guimarães, M. P. 1991. Fasciola hepatica. In: Neves, D. P. ed. Parasitologia humana. $8^{a}$. ed. São Paulo, Atheneu. p.224-229.

Hostettmann, K.; Kizu, H. \& Tomimori, T. 1982. Molluscicida properties of various saponins. Planta Medica 44:34-35.

Kloos, H. \& McCullough, F. S. 1981. Plant molluscicides: a review. Genève, World Health Organization. 32p.

1982. Plant molluscicides. Planta Medica 46:195-209.

1987. Plants with recognized molluscicidal activity. In Mотт, K. E. ed. Plant molluscicides. New York, Wiley. p. $45-108$.

Lemma, A. \& Yau, P. 1974. Studies on the molluscicidal properties of endod (Phytolacca dodecandra), III. Ethiopian Medical Journal 12:109-114.

Lemma, A.; Brody, G.; Newell, G. W.; Parkhurst, R. M. \& Skinner, W. A. 1972. Studies on the molluscicidal properties of endod (Phytolacca dodecandra): I. Increased potency with butanol extraction. Journal of Parasitology 58(1):104-107.

McCullough, F. S.; Gayral, P.; Duncan, J. \& Christie, J. D. 1980. Molluscicides in schistosomiasis control. Bulletin of the World Health Organization 58(5):681-689.

Mendes, N. M.; Pereira, J. P.; Souza, C. P. \& Azeredo, M. L. L. 1984. Ensaios preliminares em laboratório para verificar a ação moluscicida de algumas espécies da flora brasileira. Revista de Saúde Pública 18:348-354.

OMS (Organización Mundial de la Salud). 1991. Empleo inocuo de plaguicidas, Genève, OMS. 29p.

Pereira, J. P.; Souza, C. P. \& Mendes, N. M. 1978. Propriedades moluscicidas da Euphorbia cotinifolia L. Revista Brasileira de Pesquisa Médica e Biológica 11:345-351.

Pinto, C. \& Almeida, A. F. 1944. Um novo método para a profilaxia da esquistossomose mansoni. Memórias do Instituto Oswaldo Cruz 40:291-311.

Rouquayrol, M. Z.; Souza, M. P. \& Matos, F. J. A. 1973. Actividad molusquicida del Pithecelobium multiflorum. Boletin de la Oficina Sanitaria Panamericana 170:170-171.

Rouquayrol, M. Z.; Souza, M. P. \& Silva, M. J. M. 1972. Atividade moluscicida de plantas do nordeste brasileiro (III). Revista Brasileira de Farmacologia 53(5):215-220.

Silva, M. J. M.; Souza, M. P. \& Rouquayrol, M. Z. 1971. Atividade moluscicida de plantas do nordeste brasileiro II. Revista Brasileira de Farmácia 52(3):117-123.

WhO (World Health Organization). 1965. Memoranda: molluscicide screening and evaluation. Bulletin of the World Health Organization 33:567-576.

Recebido em maio de 2003. Aceito em junho de 2005. ISSN 0073-4721

Artigo disponível em: www.scielo.br/isz 